\title{
Design and Implementation of Two Degree of freedom Proportional Integral Derivative Controller
}

\author{
Dr. Raaed Faleh Hassan \\ Department of Medical Instruments Eng. Techniques \\ College of Electrical and Electronic Eng. Techniques - Middle Technical University \\ Baghdad - Iraq \\ drraaed@hotmail.com
}

\begin{abstract}
The paper presents that genetic algorithm can be used for tuning two degree of freedom Proportional Integral Derivative (2DOF - PID) controller. 3rd order plant is considered to be controlled using (2DOF - PID) controller, firstly 3rd order plant has been tested without controller in closed loop system. Secondly the system response is tested with proportional controller then with conventional PID controller, Genetic Algorithm used in both cases in order to obtain optimum response and disturbance rejection. Finally, 2DOF - PID controller is considered for controlling the above plant in closed loop system. The transfer function of this controller is mathematically rearranged in order to have three parameters to be tuned using GA. Simulation results show that 2DOF - PID controller is an effective controller for tracking command signal and disturbance rejection.
\end{abstract}

Keywords: 2DOF - PID controller, Genetic Algorithm

\section{INTRODUCTION}

Proportional - Integral - Derivative (PID) controllers are widely used as a core of industrial control applications. This popularity is mainly due to simplicity in determining its parameters and implementation in hardware and software. Despite this popularity, PID controllers cannot provide simultaneously shape the responses to both reference and disturbance signals. This is due to the fact that conventional PID controller has one closed loop transfer function, therefore, it is classified as 1DOF (one degree of freedom) controller [1].

As the PID controller is classified as 1DOF, therefore if the parameters of the PID are tuned to optimize the response of the control system to the command signal, the performance to the disturbance rejection will be deteriorated [1,2].

To solve this problem 2DOF PID controller has been considered in the literature. The dominant feature of the $2 \mathrm{DOF}$ PID controller is the two sets of parameters. One of these sets can be used to optimize the performance of the control system to the command signal, while the other sets can be used to optimize the performance against the disturbance $[1,2]$.
In [1-8], various structures of 2DOF PID controller were proposed and different tuning methods were applied in order to obtain good control performance both in command tracking and disturbance rejection characteristics.

In this paper, Genetic Algorithm GA will be considered for tuning 2DOF PID controller parameters to achieve optimum control performance for a $3^{\text {rd }}$ order plant from command tracking and disturbance rejection points of view.

\section{2DOF PID CONTROLLER}

A good performance for both set-point tracking and disturbance rejection can be achieved by using 2DOF PID controller which is also known as ISA - PID controller. The structure contains a standard PID controller in the feedback loop and a pre - filter to the command signal as shown in figure (1) [1].

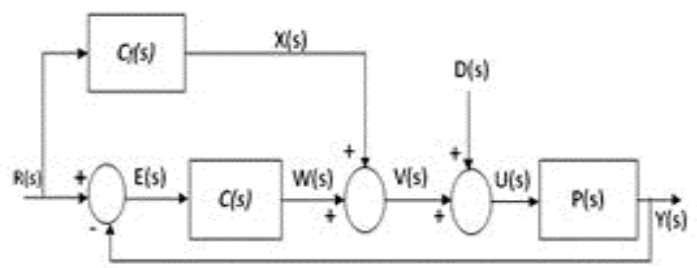




\section{FIG (1): 2DOF PID Control System}

Where $\mathrm{R}(\mathrm{s})$ is the command signal (reference input), $\mathrm{E}(\mathrm{s})$ is the error signal, $\mathrm{D}(\mathrm{s})$ is the disturbance and $\mathrm{Y}(\mathrm{s})$ is the system response.

The input - output relations of the closed - loop system is given as [1,2]:

$$
Y(s)=\frac{P(s)\left(C(s)+C_{f}(s)\right.}{1+P(s) C(s)} R(s)+\frac{P(s)}{1+P(s) C(s)} D(s)
$$

Where:

$$
C(s)=k_{p}\left(1+\frac{1}{T_{i} S}+T_{d} s\right)
$$

Which represents conventional PID controller with proportional gain $k_{p}$, integral coefficient $\left(\frac{k_{p}}{T_{i}}\right)$ and derivative coefficient $k_{p} T_{d}$.

and

$$
C_{f}(s)=k_{p}\left(\alpha+\beta T_{d} s\right)
$$

Where $\alpha$ and $\beta$ are a weighted parameters of feed forward controller, or a pre - filter.

The discrete 2DOF PID controller can be obtained by mapping equations (2) and (3) from s-domain to z-domain using bilinear transformation, therefore:

$$
\begin{aligned}
& C(z)=\left.C(s)\right|_{s=\frac{2 z-1}{T z+1}} \\
& C_{f}(z)=\left.C_{f}(s)\right|_{s=\frac{2 z-1}{T z+1}}
\end{aligned}
$$

Therefore, equation (4) will be

$$
C(z)=k_{p}\left[\frac{\left(1+\frac{2 T_{d}}{T}+\frac{T}{2 T_{i}}\right) Z^{2}+\left(\frac{T}{T_{i}}-\frac{4 T_{d}}{T}\right) Z+\left(\frac{T}{2 T_{i}}+\frac{2 T_{d}}{T}-1\right)}{Z^{2}-1}\right]
$$

While, equation (5) will be

$$
C_{f}(z)=k_{p}\left[\frac{\left(\alpha+\frac{2 \beta T_{d}}{T}\right) Z+\left(\alpha-\frac{2 \beta T_{d}}{T}\right)}{Z+1}\right]
$$

Equation (6) can be rearranged to the following form

$$
C(z)=\frac{L Z^{2}+M Z+(L-2)}{Z^{2}-1}
$$

Where: $\quad L=k_{p}\left(1+\frac{2 T_{d}}{T}+\frac{T}{2 T_{i}}\right)$

$$
M=k_{p}\left(\frac{T}{T_{i}}-\frac{4 T_{d}}{T}\right)
$$

and equation (7) can be modified to the following form

$$
C_{f}(z)=\left[\frac{N Z+(N-2)}{Z+1}\right]
$$

Where: $N=-k_{p}\left(\alpha+\frac{2 \beta T_{d}}{T}\right)$

The discrete form of the $3^{\text {rd }}$ order plant considered in this paper is:

$$
P(z)=\left(\frac{0.0001436 z^{-1}+0.0004951 z^{-2}+0.000104 z^{-3}}{1-2.464 z^{-1}+2.018 z^{-2}-0.5488 z^{-3}}\right)
$$

\section{DISCRETE - TIME CONTROL SYSTEM}

Referring to equations $(8-10)$ and fig (1), the set of LTI difference equations are shown below:

$$
\begin{aligned}
& x(n)=-x(n-1)+N r(n)+(N-2) r(n-1) \\
& w(n)=w(n-2)+\operatorname{Le}(n)+\operatorname{Me}(n-1)+(L-2) e(n-2) \\
& v(n)=x(n)+w(n) \\
& u(n)=v(n)+d(n) \\
& y(n)=2.99 y(n-1)+2.98 y(n-2)-0.99 y(n-3) \\
& +10^{-7}(1.662 x(n-1) \\
& +6.633 x(n-2 \\
& +1.654 x(n-3)) \\
& |e(n)|=|r(n)-y(n)| \\
& I A E=\sum_{k=0}^{n}|e(k)|
\end{aligned}
$$

Minimization of the IAE represents optimization of the system response to both reference signal $r(n)$ and the output disturbance rejection. To achieve this goal, there are three parameters must be tuned; these are $\mathrm{L}, \mathrm{M}$, and $\mathrm{N}$.

\section{GENETIC ALGORITHM}




\section{ICIT 2015 The $7^{\text {th }}$ International Conference on Information Technology}

doi:10.15849/icit.2015.0011 $\quad$ C ICIT 2015 (http://icit.zuj.edu.jo/ICIT15)

Genetic Algorithm is a stochastic global searching algorithm used to solve complicated problems by simulating the evolutionary course of natural selection and natural inheritance of biology circles. In genetic Algorithm, code space is used to replace problem space, fitness function is regarded as evaluating criterion, code population is regarded as evolution base, selection and genetic mechanism is actualized by genetic operation on individual bit chain of population. A repeated course is formed in this way. The individual of population evolves ceaselessly by recombining some important genes of code bit chain stochastically, and approaches to the optimal gradually till reaching the goal of solving the problem ultimately [9]. In spite of considering GA to be a robust optimization algorithm, it has some drawbacks. The main drawback is the GA cannot assure constant optimization response time, and it is unreasonable to use GA for on - line controls in real system

\section{OPTIMIZATION PROCESS}

In this section the methodology for tuning the optimization of 2DOF - PID controller is described.

Firstly, the plant in eq.(10) has been considered in closed - loop control system with proportional controller only. GA used to obtain the optimum gain for the proportional controller, in this situation the optimization process performed for tuning one parameter.

1DOF - PID controller used for controlling the plant in closed loop control system, the optimization process in this case will has three parameters to be tuned.

Finally, the set of difference equations $(11-17)$ has been implemented as a Matlab function file in order to calculate the fitness function which is Integral Absolute Error (IAE). The task of GA algorithm is to minimize this fitness function by selecting the three unknowns $\mathrm{L}, \mathrm{M}$ and $\mathrm{N}$.

\section{SIMULATION RESULTS}

Fig (2) shows the response of the closed loop system of the $3^{\text {rd }}$ order plant without controller. From his figure it can be seen that the system response isn't track the command signal and there is significant error.
Genetic Algorithm has been used to determine the optimum gain needed to minimize the error between the command signal and the system response.

Fig (3) shows that only adding gain to the system isn't sufficient because this gain produces high peak overshoot (more than $20 \%$ ) and a hard oscillation.

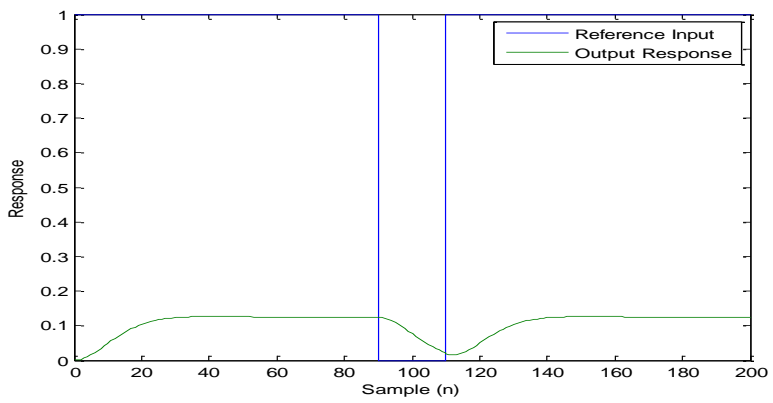

FIG (2): $3^{\text {rd }}$ order plant without controller

The results of optimization process using GA are summarized in table 1

\section{Table 1}

\begin{tabular}{|c|c|c|}
\hline No. of Generation & No. of evaluation & Best fitness \\
\hline 292 & 586000 & 41.4932 \\
\hline
\end{tabular}

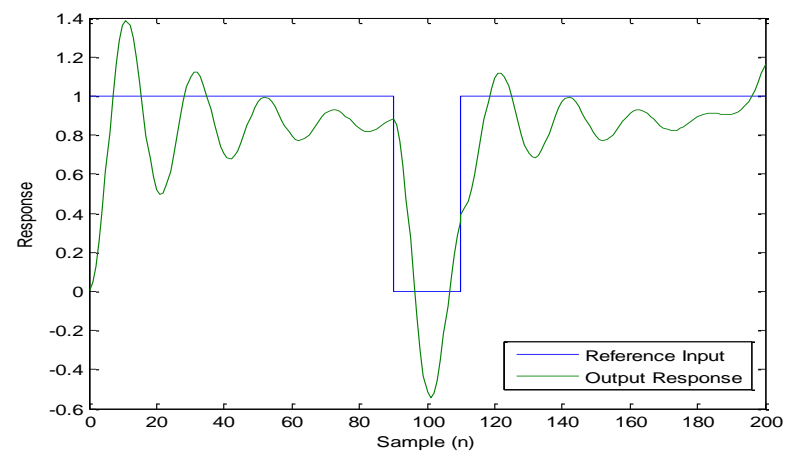

FIG (3): Closed Loop control system with proportional controller. 
According to the previous results and in order to overcome the challenges appear, 1 DOF - PID controller has been considered.

The task of GA is to tune three parameters $k_{p}, k_{i} \& k_{d}$ of the PID controller for obtaining the optimum response to the command signal.

Fig (4) shows system response to the command signal. The result indicates that the response has very high peak overshoot and steady state error.

The results of optimization process are summarized in table 2 .

Table 2

\begin{tabular}{|c|c|c|}
\hline No. of Generation & No. of evaluation & Best fitness \\
\hline 1000 & 2002000 & 49.2411 \\
\hline
\end{tabular}

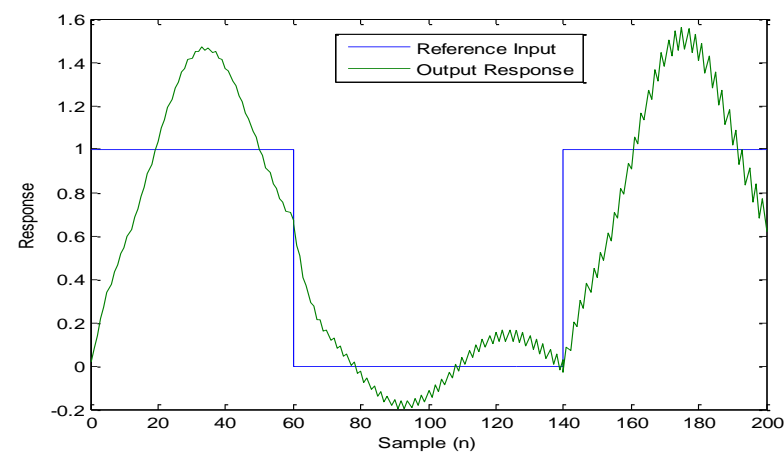

FIG (4): Closed Loop control system with 1DOF - PID controller.

Now, conventional PID controller has been tested under the presence of output disturbance. Fig (5) shows the system response for unit step input and finite duration of output disturbance. From these results, it can be noticed that the control system has acceptable disturbance rejection but tracking of the command signal still has high peak overshoot.

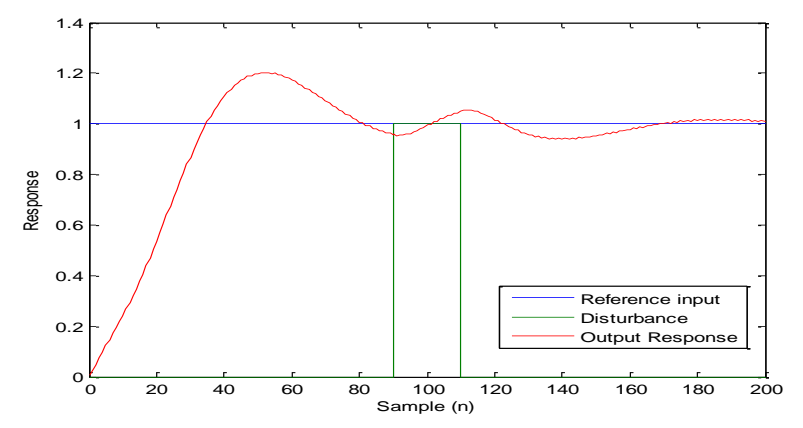

FIG (5): Disturbance rejection of closed loop control system with 1DOF PID Controller

The optimization process results are summarized in table 3 .

Table 3

\begin{tabular}{|c|c|c|}
\hline No. of Generation & No. of evaluation & Best fitness \\
\hline 1000 & 2002000 & 49.2411 \\
\hline
\end{tabular}

2DOF PID controller is considered in order to improve system response for tracking the command signal and disturbance rejection. Fig (6) shows simulation results of the system response to the command signal.

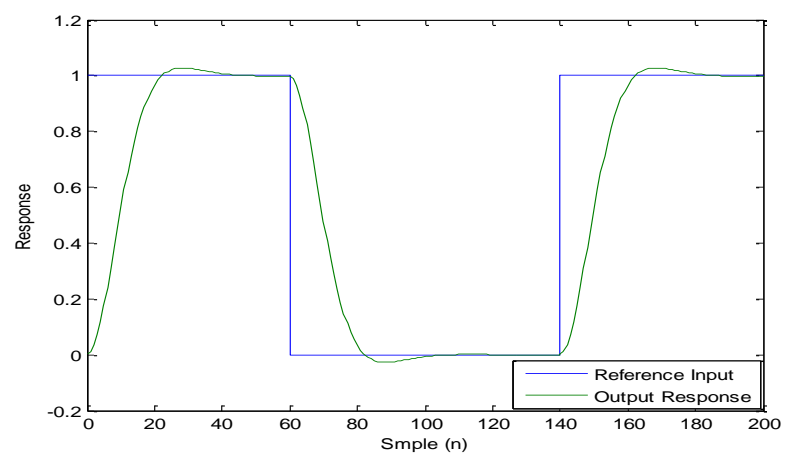

FIG (6): Closed Loop control system with 2DOF - PID controller.

The optimization process results are summarized in table 4 .

Table 4

\begin{tabular}{|c|c|c|}
\hline No. of Generation & No. of evaluation & Best fitness \\
\hline 235 & 472000 & 32.2484 \\
\hline
\end{tabular}




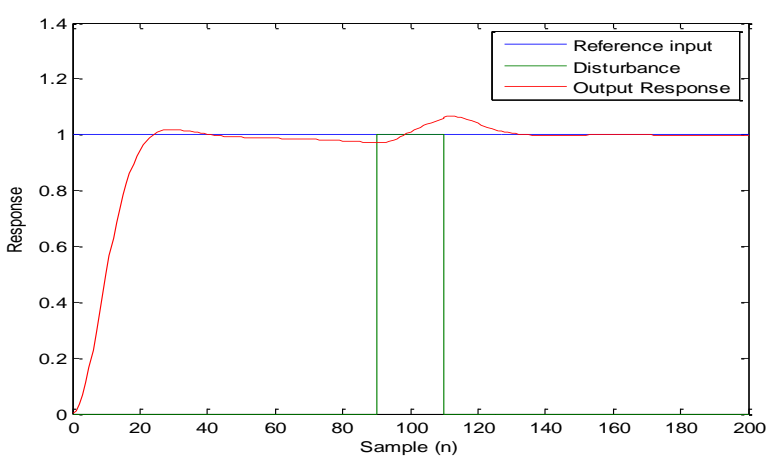

FIG (7): Disturbance rejection of closed loop control system with 2DOF PID Controller

2DOF PID controller is also considered for controlling the plant to achieve both command signal tracking and output disturbance rejection.

Fig (7) shows simulation results of this situation, it is clear from these results that the using of 2DOF PID controller improved the performance of the control system for tracking command signal and output disturbance rejection.

The results of optimization process are summarized in table 5 .

Table 5

\begin{tabular}{|c|c|c|}
\hline No. of Generation & No. of evaluation & Best fitness \\
\hline 291 & 584000 & 12.4443 \\
\hline
\end{tabular}

Table 6 shows the performance summary of different controllers.

Table 6

\begin{tabular}{|l|c|c|}
\hline \multirow{2}{*}{$\begin{array}{l}\text { Type of } \\
\text { Controller }\end{array}$} & \multicolumn{2}{|c|}{ Fitness function (IAE) } \\
\cline { 2 - 3 } & $\begin{array}{c}\text { Command } \\
\text { Signal }\end{array}$ & $\begin{array}{c}\text { Disturbance } \\
\text { Rejection }\end{array}$ \\
\hline Proportional $\mathrm{K}_{\mathrm{p}}$ & 41.4932 & -------- \\
\hline 1DOF PID & 49.2411 & 27.8699 \\
\hline 2DOF PID & 32.2484 & 12.4443 \\
\hline
\end{tabular}

VII. CONCLUSIONS
In this paper genetic algorithm has been used as an optimization tools for tuning different controller structures for controlling $3^{\text {rd }}$ order plant. Proportional, conventional PID and 2DOF - PID controllers are considered for controlling the above plant. The parameters of these controllers are tuned using genetic algorithm and from simulation results it can be seen that only 2DOF - PID controller able to make the system to keep track of the command signal as well as reject the output disturbance.

\section{REFERENCES}

[1] Mituhiko Araki and Hidefuni Taguchi "Two - Degree - of - Freedom PID controllers", International Journal of Control, Automation and Systems, Vol. 1 No. 4, December 2003.

[2] Jing - Gang Zhang, Zhi -yvan Liu and Run Pei “ Two - Dgree - of - Freedom PID control with Fuzzy Logic Compensation", proceeding of the first International conference on machine learning and Cybernetics, Beijing, 4 - 5 November 2002.

[3] Takao Sato, Akira Inoue and Toru Yamamoto " Two - Dgree - of - Freedom PID controller based on Extended Generalized Minimum Variance Control" International Journal of Innovative computing Information and Control, Vol. 4, No. 12, December 2008.

[4] Gish Herjolfsson, Anna Soffia Hauksdottir and Sven P. Sigurdsson “ A Two - Stage optimization PID Algorithm" IFAC conference in PID control, Brescia (Italy), March 28 - 30, 2012.

[5] Eiji Takegami, Kohji Higuchi, Kazushi Nakano, et. al." The Method for Determining Parameters of Approximate 2DOF Digital Controller for Robust Control of DC - DC Converter" ECTI Transactions on Electrical Eng., Electronics and communications, Vol.4. No.1, February 2006.

[6] Tohru Kawabe " Robust 2DOF PID controller Design of Time - Delay Systems Based on Evolutionary computation" $4^{\text {th }}$ WSEAS International conference on Electronics, Control and signal Processing, Miami, Florida, USA, 17 - 19 November 2005 (pp 144 - 149).

[7] Humberto M. Mazzini, Fabio G. Dos Santos “ Two Degree - of - Freedom PID Control for Integrating process" XVIII congress, Brazil, 12 - 16 September 2010. 
[8] Takao Sato, Akira Inoue and Yoichi Hirashima, "SelfTuning Two-Degree-of-Freedom PID Controller Reducing the Effect of Disturbances" Proceedings of the American Control Conference Anchorage, AK May 8-10, 2002.

[9] Tai-Shan Yan, Yong-Qing Tao, and Du-Wu Cui, “ Research on Handwritten Numeral Recognition Method Based on Improved Genetic Algorithm and Neural Network", Proceedings of the 2007 International Conference on Wavelet Analysis and Pattern Recognition, Beijing, China, 2-4 Nov. 2007. 